\title{
Some fixed point theorems in Menger probabilistic metric-like spaces
}

\author{
Antonio Francisco Roldán López de Hierro ${ }^{*^{*}}$ (1) and Manuel de la Sen ${ }^{2}$
}

\author{
"Correspondence: aroldan@ugr.es \\ ${ }^{1}$ Department of Quantitative \\ Methods for Economics and \\ Business, Faculty of Economics and \\ Business Sciences, University of \\ Granada, Campus Universitario de \\ Cartuja, s/n, Granada, 18011, Spain \\ Full list of author information is \\ available at the end of the article
}

\begin{abstract}
In this manuscript we extend very recent fixed point theorems in the setting of Menger spaces in three senses: on the one hand, we introduce the notion of Menger probabilistic metric-like space by avoiding a non-necessary constraint (at least, for our purposes) in the properties that define a Menger space; on the other hand, we consider a more general class of auxiliary functions in the contractivity condition; finally, we show that the function $t \rightarrow 1 / t-1$ (which appears in many fixed point theorems in the fuzzy context) can be replaced by more appropriate and general functions. We illustrate our main statements with an example in which previous results cannot be applied.
\end{abstract}

Keywords: probabilistic metric space; Menger space; fixed point; contractive mapping

\section{Introduction}

There are two well known extensions of the notion of metric space to frameworks in which imprecise models are considered: fuzzy metric spaces (see [1-7]) and probabilistic metric spaces [8-11]. The two concepts are very similar, but they are different in nature.

In a metric space, the distance between two points is, necessarily, a non-negative real number. In 1942, Menger extended the notion of metric space replacing non-negative numbers by a random variable that only took non-negative real values. Such random variable was characterized by its distribution function. Hence, in this kind of spaces (which Menger originally called statistical metric spaces), the distance between two points $x$ and $y$ is given by a distribution function $F_{x y}$ which can be seen as follows: ${ }^{\mathrm{a}}$ for all $t \in[0, \infty)$, the value $F_{x y}(t)$ gives the probability of the event that occurs when the distance between $x$ and $y$ is less than $t$, that is,

$$
F_{x y}(t)=P(d(x, y)<t) \quad \text { for all } t \in[0, \infty)
$$

Thus, statistical metric spaces depend on the notion of distribution function. Although there is a great agreement about the global properties that a distribution function must satisfy, in practice, we can find several papers involving different notions. Such different definitions lead to different properties and, in some cases, to incomparable results. For instance, Schweizer and Sklar [8], Definition 4.1.1, established that a distribution function (briefly, d.f.) is a nondecreasing function $F: \overline{\mathbb{R}} \rightarrow[0,1]$ such that $F(-\infty)=0$ and $F(\infty)=1$

(c) 2015 Roldán López de Hierro and de la Sen. This article is distributed under the terms of the Creative Commons Attribution 4.0 International License (http://creativecommons.org/licenses/by/4.0/), which permits unrestricted use, distribution, and reproduction in any medium, provided you give appropriate credit to the original author(s) and the source, provide a link to the Creative Commons license, and indicate if changes were made. 
(where $\overline{\mathbb{R}}=[-\infty, \infty]$ is the extended real line). Note that not all such functions can be interpreted as cumulative distribution functions for a random variable. However, when a random experiment is considered, additional properties are required. For instance, if $F$ is given as in (1), it is not difficult to show that $F$ is left-continuous in $\mathbb{R}=(-\infty, \infty)$. Furthermore, if $d$ only takes non-negative values, then $F(t)=0$ for all $t \in[-\infty, 0]$. Thus, Schweizer and Sklar denoted by $\Delta$ the family of all d.f's that are left-continuous on $\mathbb{R}$, and by $\Delta^{+}$the family of all $F \in \Delta$ such that $F(t)=0$ for all $t \in[-\infty, 0]$. Functions in $\Delta^{+}$ were called distance distribution functions (briefly, d.d.f.) because they represent random variables that only take non-negative values (as in (1), where a distance is involved). For convenience, d.d.f.'s are only considered in $[0, \infty]$, where $F(0)=0$ and $F(\infty)=1$.

The set $\Delta^{+}$of all d.d.f.s is partially ordered by the binary relation $\leq$, being $F \leq G$ when $F(t) \leq G(t)$ for all $t \in(0, \infty)$. The minimal and the maximal elements of $\Delta^{+}$are $\epsilon_{\infty}$ and $\epsilon_{0}$, respectively, where

$$
\epsilon_{\infty}(t)=\left\{\begin{array}{ll}
0, & \text { if } 0 \leq t<\infty, \\
1, & \text { if } t=\infty,
\end{array} \text { and } \quad \epsilon_{0}(t)= \begin{cases}0, & \text { if } t=0 \\
1, & \text { if } 0<t \leq \infty\end{cases}\right.
$$

On the other hand, many authors call distribution function (see, for instance, [12]) to a nondecreasing, left-continuous function $F: \mathbb{R} \rightarrow[0,1]$ such that $\inf _{t \in \mathbb{R}} F(t)=0$ and $\sup _{t \in \mathbb{R}} F(t)=1$. As $F$ is nondecreasing, it follows that

$$
\lim _{t \rightarrow-\infty} F(t)=\inf _{t \in \mathbb{R}} F(t)=0 \quad \text { and } \quad \lim _{t \rightarrow \infty} F(t)=\sup _{t \in \mathbb{R}} F(t)=1
$$

This notion does not coincide with Schweizer and Sklar's view-point. For instance, $\varepsilon_{\infty}$ is a d.d.f. in Schweizer and Sklar's sense, but it does not satisfy the condition $\lim _{t \rightarrow \infty} F(t)=1$ (because if $t \in[0, \infty)$, then $F(t)=0)$.

In recent times, many fixed point theorems have been presented in the setting of probabilistic metric spaces. Many of them were inspired by their corresponding results on metric spaces. One of the most attractive, effective ways to introduce contractivity conditions in the probabilistic framework is based on considering some terms like in the following expression:

$$
\frac{1}{F_{x y}(t)}-1, \quad \text { where } x, y \in X \text { and } t>0
$$

(see [13-17]). For instance, in [12], Kutbi et al. stated the following result (where $\Phi$ and $\Psi_{0}$ are appropriate collections of auxiliary functions that we will describe in Section 2).

Theorem 1 (Kutbi et al. [12], Theorem 2.1) Let $(X, F, T)$ be a G-complete Menger space and let $f: X \rightarrow X$ be a mapping. Assume that there exist a constant $c \in(0,1)$ and two functions $\phi \in \Phi$ and $\psi \in \Psi_{0}$ satisfying the inequality

$$
\frac{1}{F_{f x, f y}(\phi(c t))}-1 \leq \psi\left(\frac{1}{F_{x, y}(\phi(t))}-1\right)
$$

for all $x, y \in X$ and all $t>0$ such that $F_{x, y}(\phi(t))>0$. Then f has a unique fixed point. 
We must highlight that the uniqueness of the fixed point was not proved in such result (it was studied in Theorem 2.2 in [12]). The main aims of the present manuscript are the following ones:

(1) to extend the previous result to a wider class of Menger spaces, that we shall call Menger probabilistic metric-like spaces;

(2) to show that some conditions in the functions of the family $\Phi$ are not necessary;

(3) to introduce more general contractivity conditions replacing the function $t \rightarrow 1 / t-1$ by an appropriate function $h$.

For the sake of clarity, we advise the reader that we shall use the terminology introduced in [8].

\section{Preliminaries}

In this section, we recall some definitions and basic results. Throughout this manuscript, let $\mathbb{N}=\{0,1,2, \ldots\}$ be the set of all non-negative integer numbers and let $\mathbb{N}^{*}=\mathbb{N} \backslash\{0\}$. A fixed point of a self-mapping $T: X \rightarrow X$ is a point $x \in X$ such that $T x=x$. We will denote by $\operatorname{Fix}(T)$ the family of all fixed points of $T$.

Definition 2 A $t$-norm (or a triangular norm) is a function $*:[0,1] \times[0,1] \rightarrow[0,1]$ satisfying, for all $a, b, c, d \in[0,1]$, the following properties:

(1) Associativity: $(a * b) * c=a *(b * c)$.

(2) Commutativity: $a * b=b * a$.

(3) Existence of unity: $a * 1=a$.

(4) Monotonicity: if $a \leq b$ and $c \leq d$, then $a * c \leq b * d$.

A $t$-norm is continuous if it is continuous as a function.

It can be proved that $a * 0=0$ for all $a \in[0,1]$. Examples of $t$-norms are:

$$
\begin{aligned}
& \text { Minimal } t \text {-norm: } a *_{M} b=\min (a, b), \\
& \text { Product } t \text {-norm: } a *_{P} b=a b, \\
& \text { Lukasiewicz } t \text {-norm: } a *_{L} b=\max (a+b-1,0), \\
& \text { Drastic } t \text {-norm: } a *_{D} b= \begin{cases}\min (a, b), & \text { if } \max (a, b)=1, \\
0, & \text { otherwise. }\end{cases}
\end{aligned}
$$

The three first $t$-norms are continuous, but the drastic $t$-norm is not continuous.

Given $a \in[0, \infty)$, let $\epsilon_{a}:[0, \infty] \rightarrow[0,1]$ be the function given by

$$
\epsilon_{a}(t)= \begin{cases}0, & \text { if } 0 \leq t \leq a \\ 1, & \text { if } a<t \leq \infty\end{cases}
$$

Functions $\left\{\epsilon_{a}: a \in[0, \infty)\right\} \cup\left\{\epsilon_{\infty}\right\}$ are called step functions and they are examples of d.d.f.s. [18].

Definition 3 A Menger probabilistic metric space (briefly, a Menger PMS) is a triple $(X, F, *)$ where $X$ is a nonempty set, $*$ is a continuous $t$-norm and $F: X \times X \rightarrow \Delta^{+}$is a mapping satisfying, for all $x, y, z \in X$ and all $t, s \in(0, \infty)$, the following properties (for convenience, the value $F(x, y)(t)$ will be denoted by $\left.F_{x y}(t)\right)$ : 
$\left(\mathrm{PM}_{1}\right) F_{x x}(t)=1$ for all $t>0$;

$\left(\mathrm{PM}_{2}\right)$ identity: if $F_{x y}(t)=1$ for all $t>0$, then $x=y$;

$\left(\mathrm{PM}_{3}\right)$ symmetry: $F_{x y}(t)=F_{y x}(t)$;

$\left(\mathrm{PM}_{4}\right)$ triangular inequality: $F_{x z}(t+s) \geq F_{x y}(t) * F_{y z}(s)$.

This definition of a Menger PMS is taken from Schweizer and Sklar's notion given in [8]. However, it is different from the one we find in [12], Definition 1.3, because the authors of this reference assumed that $F_{x y}$ is a distribution function in their own sense (in particular, the random variable can take values on intervals of negative real numbers).

Remark 4 The reader can observe the following subtle details.

(1) Since $F(X \times X) \subseteq \Delta^{+}$, each d.d.f. $F_{x y}$ in a Menger PMS is a nondecreasing function on $[0, \infty]$. Furthermore, it always satisfies $F_{x y}(0)=0$. In the following text, we will often use the monotonicity of $F_{x y}$. However, we will never use the fact that $F_{x y}(0)=0$. In this sense, it is possible to consider a similar definition of Menger space where the probabilistic metric $F$ take values in $\Delta$ rather than in $\Delta^{+}$. However, notice that conditions $\left(\mathrm{PM}_{1}\right)-\left(\mathrm{PM}_{4}\right)$ only refer to the interval $[0, \infty)$.

(2) In the field of fixed point theory, a fifth assumption is usually considered.

$\left(\mathrm{PM}_{5}\right) \lim _{t \rightarrow \infty} F_{x y}(t)=1$ for all $x, y \in X$.

A discussion about the necessity of this condition can be found in [19]. Our main result will not need such property, but it will appear in some corollaries.

The following families of auxiliary functions were considered in [12].

Definition 5 Let $\Phi$ be the family of all functions $\phi:[0, \infty) \rightarrow[0, \infty)$ satisfying:

(1) $\phi(t)=0$ if, and only if, $t=0$;

(2) $\phi$ is strictly increasing and $\lim _{t \rightarrow \infty} \phi(t)=\infty$;

(3) $\phi$ is left-continuous in $(0, \infty)$;

(4) $\phi$ is continuous at $t=0$.

Definition 6 Let $\Psi_{0}$ be the class of all functions $\psi:[0, \infty) \rightarrow[0, \infty)$ satisfying:

(1) $\psi$ is nondecreasing;

(2) $\psi$ is continuous at $t=0$;

(3) $\psi(0)=0$;

(4) if $\left\{a_{n}\right\} \subset[0, \infty)$ is a sequence such that $\left\{a_{n}\right\} \rightarrow 0$, then $\left\{\psi^{n}\left(a_{n}\right)\right\} \rightarrow 0$ (where $\psi^{n}$ denotes the $n$ th-iterate of $\psi$ ).

First of all, we show that we do not need to assume that $\psi$ is continuous at $t=0$ for functions in $\Psi_{0}$ under the rest of the assumptions.

Proposition 7 Let $\psi:[0, \infty) \rightarrow[0, \infty)$ be a nondecreasing function such that $\psi(0)=0$.

(1) If $\psi$ is not continuous at $t=0$, then there exists $\varepsilon_{0}>0$ such that $\psi(t) \geq \varepsilon_{0}$ for all $t>0$.

(2) If $\psi$ satisfies ' $\left\{\psi^{n}\left(a_{n}\right)\right\} \rightarrow 0$ whenever $\left\{a_{n}\right\} \rightarrow 0$ as $n \rightarrow \infty$ ', then $\psi$ is continuous at $t=0$. 
Proof (1) Taking into account that $\psi(0)=0$, then the continuity condition at $t=0$ is

$$
\forall \varepsilon>0, \exists \delta>0:(|t-0|<\delta \Rightarrow|\psi(t)-\psi(0)|<\varepsilon) .
$$

Assume that $\psi$ is not continuous at $t=0$. Then there exists $\varepsilon_{0}>0$ verifying

for all $\delta>0$, there exists $t \in(0, \delta)$ such that $\psi(t) \geq \varepsilon_{0}$.

By taking $\delta_{n}=1 / n>0$ for all $n \in \mathbb{N}$, we can find a strictly decreasing sequence $\left\{a_{n}\right\} \subset(0, \infty)$ such that

$$
0<a_{n+1}<a_{n}<\frac{1}{n} \quad \text { and } \quad \psi\left(a_{n}\right) \geq \varepsilon_{0} \quad \text { for all } n \in \mathbb{N} .
$$

Next, we show that $\psi(t) \geq \varepsilon_{0}$ for all $t>0$. Let $t>0$ be arbitrary. As $\left\{a_{n}\right\} \rightarrow 0$, there exists $n_{0} \in \mathbb{N}$ such that $0<a_{n_{0}}<t$. As $\psi$ is nondecreasing,

$$
\psi(t) \geq \psi\left(a_{n_{0}}\right) \geq \varepsilon_{0} .
$$

Then $\psi(t) \geq \varepsilon_{0}$.

(2) We reason by contradiction. Assume that $\psi$ is not continuous at $t=0$. By the first item, there exists $\varepsilon_{0}>0$ such that $\psi(t) \geq \varepsilon_{0}$ for all $t>0$. Let $a_{n}=1 / n$ for all $n \in \mathbb{N}$. Then $\left\{a_{n}\right\} \rightarrow 0$. By hypothesis, $\left\{\psi^{n}\left(a_{n}\right)\right\} \rightarrow 0$. However, for all $n \in \mathbb{N}$,

$$
\begin{aligned}
& a_{n}=\frac{1}{n}>0 \quad \Rightarrow \quad \psi\left(a_{n}\right) \geq \varepsilon_{0}>0 \\
& \psi\left(a_{n}\right)>0 \quad \Rightarrow \quad \psi^{2}\left(a_{n}\right) \geq \varepsilon_{0}>0 \\
& \vdots \\
& \psi^{n-1}\left(a_{n}\right)>0 \quad \Rightarrow \quad \psi^{n}\left(a_{n}\right)=\psi\left(\psi^{n-1}\left(a_{n}\right)\right) \geq \varepsilon_{0} .
\end{aligned}
$$

Since $\psi^{n}\left(a_{n}\right) \geq \varepsilon_{0}$ for all $n \in \mathbb{N},\left\{\psi^{n}\left(a_{n}\right)\right\} \rightarrow 0$ is a contradiction.

As a consequence of the previous properties, we can express

$$
\Psi_{0}=\left\{\psi:[0, \infty) \rightarrow[0, \infty) / \begin{array}{l}
\psi \text { nondecreasing, } \psi(0)=0, \\
\text { if }\left\{a_{n}\right\} \rightarrow 0 \text {, then }\left\{\psi^{n}\left(a_{n}\right)\right\} \rightarrow 0 .
\end{array}\right\}
$$

\section{Menger probabilistic metric-like spaces}

In this section, we introduce the notion of Menger probabilistic metric-like space as a natural way to extend Menger PM-spaces.

Definition 8 A Menger probabilistic metric-like space (briefly, a Menger PMLS) is a triple $(X, F, *)$ where $X$ is a nonempty set, $*$ is a continuous $t$-norm and $F: X \times X \rightarrow \Delta^{+}$is a mapping satisfying $\left(\mathrm{PM}_{2}\right),\left(\mathrm{PM}_{3}\right)$, and $\left(\mathrm{PM}_{4}\right)$.

Clearly, every Menger PMS is a Menger PMLS, but the converse is false, as we can see in the following example. 
Example 9 Let $X=[0, \infty)$ be the interval of non-negative real numbers and let $F: X \times$ $X \rightarrow \Delta^{+}$be the mapping:

$$
F_{x y}=\epsilon_{\max \{x, y\}} \quad \text { for all } x, y \in X,
$$

where $\left\{\epsilon_{a}: a \in[0, \infty)\right\}$ are the step functions given in (2). Let us show that $\left(X, F, *_{P}\right)$ is a Menger PMLS that also verifies $\left(\mathrm{PM}_{5}\right)$, but it is not a Menger PMS. Indeed, since $F_{22}(1)=$ $\epsilon_{2}(1)=0$, then $F$ does not satisfy axiom $\left(\mathrm{PM}_{1}\right)$, so $\left(X, F, *_{P}\right)$ is not a Menger PMS. However, conditions $\left(\mathrm{PM}_{2}\right),\left(\mathrm{PM}_{3}\right)$, and $\left(\mathrm{PM}_{5}\right)$ are obvious. Let us prove condition $\left(\mathrm{PM}_{4}\right)$. Let $x, y, z \in$ $X$ and $t, s>0$. Since $F_{x z}(t+s) \in\{0,1\},\left(\mathrm{PM}_{4}\right)$ trivially holds if $F_{x z}(t+s)=1$. Assume that $F_{x z}(t+s)=0$. Hence $\epsilon_{\max \{x, z\}}(t+s)=0$ implies that $t+s \leq \max \{x, z\}$. Then $t+s \leq x$ or $t+s \leq z$. In the first case, $t \leq t+s \leq x$, so $F_{x y}(t)=\epsilon_{\max \{x, y\}}(t)=0$. In the second case, $s \leq$ $t+s \leq z$, so $F_{y z}(s)=\epsilon_{\max \{y, z\}}(s)=0$. In any case, $0 \in\left\{F_{x y}(t), F_{y z}(s)\right\}$. Then

$$
F_{x y}(t) *_{P} F_{y z}(s)=F_{x y}(t) \cdot F_{y z}(s)=0=F_{x z}(t+s),
$$

and this proves that $\left(\mathrm{PM}_{4}\right)$ also holds. Thus, $\left(X, F, *_{P}\right)$ is a Menger PMLS that also verifies $\left(\mathrm{PM}_{5}\right)$.

Example 10 Using $X$ and $F$ as in the previous example, and taking into account that $a *_{P} b \leq a *_{M} b$ for all $a, b \in[0,1]$, we deduce that $\left(X, F, *_{M}\right)$ is also a Menger PMLS that, additionally, verifies $\left(\mathrm{PM}_{5}\right)$, but it is not a Menger PMS.

Definition 11 Let $\left\{x_{n}\right\}$ be a sequence in a Menger PMLS $(X, F, *)$. We will say that:

- $\left\{x_{n}\right\}$ converges to $x$ if for all $\varepsilon>0$ and all $\lambda \in(0,1)$, there exists $n_{0} \in \mathbb{N}$ such that $F_{x_{n} x}(\varepsilon)>1-\lambda$ for all $n \geq n_{0}$ (in such a case, we will write $\left\{x_{n}\right\} \rightarrow x$ );

- $\left\{x_{n}\right\}$ is a Cauchy sequence if for all $\varepsilon>0$ and all $\lambda \in(0,1)$, there exists $n_{0} \in \mathbb{N}$ such that $F_{x_{n} x_{m}}(\varepsilon)>1-\lambda$ for all $m>n \geq n_{0}$;

- $(X, F, *)$ is $M$-complete if every Cauchy sequence is convergent;

- $\left\{x_{n}\right\}$ is a G-Cauchy sequence if for all $\varepsilon>0$ and all $p \in \mathbb{N}^{*}$, we have $\lim _{n \rightarrow \infty} F_{x_{n} x_{n+p}}(\varepsilon)=1$

- $(X, F, *)$ is $G$-complete if every $G$-Cauchy sequence is convergent.

Notice that the $t$-norm $*$ has not a role in the previous definition. It is clear that every Cauchy sequence is also a $G$-Cauchy sequence. As a consequence, every G-complete Menger PMLS is an $M$-complete Menger PMLS, but the converse is false.

Example 12 Let us show that the Menger PMLS $\left(X, F, *_{P}\right)$ introduced in Example 9 is $G$ complete. Let $\left\{x_{n}\right\}$ be a $G$-Cauchy sequence in $\left(X, F, *_{P}\right)$. We claim that $\left\{x_{n}\right\} \stackrel{d}{\rightarrow} 0$ using the Euclidean metric $d(x, y)=|x-y|$ for all $x, y \in X$. Let $\varepsilon>0$ be arbitrary. Using $p=1$, we have $\lim _{n \rightarrow \infty} F_{x_{n} x_{n+1}}(\varepsilon)=1$. As $F_{x_{n} x_{n+1}}(\varepsilon) \in\{0,1\}$ for all $n \in \mathbb{N}$, we infer that there exists $n_{0} \in \mathbb{N}$ such that $F_{x_{n} x_{n+1}}(\varepsilon)=1$ for all $n \geq n_{0}$. Hence

$$
1=F_{x_{n} x_{n+1}}(\varepsilon)=\epsilon_{\max \left\{x_{n}, x_{n+1}\right\}}(\varepsilon) \quad \Rightarrow \quad \max \left\{x_{n}, x_{n+1}\right\}<\varepsilon .
$$


Then $x_{n} \leq \max \left\{x_{n}, x_{n+1}\right\}<\varepsilon$ for all $n \geq n_{0}$. This proves that $\left\{x_{n}\right\} \stackrel{d}{\rightarrow} 0$. Next, we are going to prove that $\left\{x_{n}\right\}$ converges to 0 in $\left(X, F, *_{P}\right)$. Indeed, notice that

$$
F_{x_{n}, 0}(\varepsilon)=\epsilon_{\max \left\{x_{n}, 0\right\}}(\varepsilon)=\epsilon_{x_{n}}(\varepsilon)= \begin{cases}0, & \text { if } \varepsilon \leq x_{n}, \\ 1, & \text { if } x_{n}<\varepsilon .\end{cases}
$$

As $\left\{x_{n}\right\} \stackrel{d}{\rightarrow} 0$, given $\varepsilon>0$, there exists $n_{0} \in \mathbb{N}$ such that $x_{n}<\varepsilon$ for all $n \geq n_{0}$. In particular,

$$
F_{x_{n}, 0}(\varepsilon)=\epsilon_{x_{n}}(\varepsilon)=1>1-\lambda
$$

for all $n \geq n_{0}$, whatever $\lambda \in(0,1)$. Thus, $\left\{x_{n}\right\}$ converges to 0 in $\left(X, F, *_{P}\right)$ and this space is G-complete.

In a Menger PMLS $(X, F, *)$, the subsets

$$
N_{\varepsilon, \lambda}(x)=\left\{y \in X: F_{x y}(\varepsilon)>1-\lambda\right\}
$$

do not determine a topology on $X$ because we cannot ensure that $x \in N_{\varepsilon, \lambda}(x)$ since axiom $\left(\mathrm{PM}_{1}\right)$ was avoided. However, we are going to show that the limit of a convergent sequence in a Menger PMLS is unique.

Lemma 13 If $\left\{x_{n}\right\}$ is a sequence in a Menger PMLS $(X, F, *)$ and $x, y \in X$ are such that $\left\{x_{n}\right\} \rightarrow x$ and $\left\{x_{n}\right\} \rightarrow y$, then $x=y$.

Proof Let $t>0$ be arbitrary. As $\left\{x_{n}\right\} \rightarrow x$ and $\left\{x_{n}\right\} \rightarrow y$, using $\varepsilon=t / 2>0$ and $\lambda_{k}=\frac{1}{k} \in(0,1)$ for all $k \in \mathbb{N}, k \geq 2$, there exists $x_{n(k)}$ such that

$$
F_{x, x_{n(k)}}\left(\frac{t}{2}\right)>1-\frac{1}{k+2} \quad \text { and } \quad F_{x_{n(k)}, y}\left(\frac{t}{2}\right)>1-\frac{1}{k} .
$$

Therefore,

$$
F_{x y}(t) \geq F_{x, x_{n(k)}}\left(\frac{t}{2}\right) * F_{x_{n(k)}, y}\left(\frac{t}{2}\right) \geq\left(1-\frac{1}{k}\right) *\left(1-\frac{1}{k}\right) .
$$

Letting $k \rightarrow \infty$ and taking into account that $*$ is continuous, we observe that

$$
F_{x y}(t) \geq \lim _{k \rightarrow \infty}\left(1-\frac{1}{k}\right) *\left(1-\frac{1}{k}\right)=1 * 1=1
$$

As a consequence, $F_{x y}(t)=1$ for all $t>0$, so $x=y$ by virtue of $\left(\mathrm{PM}_{2}\right)$.

The following result characterizes the Menger PMS as a particular subclass of Menger PMLS.

Lemma 14 If $(X, F, *)$ is a Menger PMLS, then the following conditions are equivalent.

(1) $(X, F, *)$ is a Menger PMS.

(2) If $\left\{x_{n}\right\} \subseteq X$ is a constant sequence and $x_{n}=z$ for all $n \in \mathbb{N}$, then $\left\{x_{n}\right\}$ converges to $z$. 
Proof Assume that $(X, F, *)$ is a Menger PMS and let $x_{n}=z \in X$ for all $n \in \mathbb{N}$. Then, for all $\varepsilon>0$ and all $\lambda \in(0,1)$,

$$
F_{x_{n}, z}(\varepsilon)=F_{z, z}(\varepsilon)=1>1-\lambda \quad \text { for all } n \in \mathbb{N} \text {. }
$$

Conversely, assume the second condition and let $x \in X$ be arbitrary. Let define $x_{n}=x$ for all $n \in \mathbb{N}$. By hypothesis, $\left\{x_{n}\right\} \rightarrow x$. Let $\varepsilon>0$ and let $\lambda_{k}=1 / k$ for all $k \in \mathbb{N}$. Then there exists $x_{n(k)}$ such that $F_{x x}(\varepsilon)=F_{x_{n(k), x}}(\varepsilon)>1-\lambda_{k}=1-1 / k$. Letting $k \rightarrow \infty$, we deduce that $F_{x x}(\varepsilon)=1$ for all $\varepsilon>0$, so assumption $\left(\mathrm{PM}_{1}\right)$ holds.

Lemma 15 If $\left\{x_{n}\right\}$ is a sequence in a Menger PMLS $(X, F, *)$ such that

$$
\lim _{n \rightarrow \infty} F_{x_{n}, x_{n+1}}(t)=1 \quad \text { for all } t>0,
$$

then $\left\{x_{n}\right\}$ is a G-Cauchy sequence in $(X, F, *)$.

Proof Let $n, p \in \mathbb{N}^{*}$ be arbitrary and let $\varepsilon>0$. We observe that

$$
F_{x_{n}, x_{n+p}}(\varepsilon) \geq F_{x_{n}, x_{n+1}}\left(\frac{\varepsilon}{p}\right) * F_{x_{n+1}, x_{n+2}}\left(\frac{\varepsilon}{p}\right) * F_{x_{n+2}, x_{n+3}}\left(\frac{\varepsilon}{p}\right) * \cdots * F_{x_{n+p-1}, x_{n+p}}\left(\frac{\varepsilon}{p}\right) .
$$

If $\varepsilon$ and $p$ are fixed, but $n \rightarrow \infty$, we deduce, taking into account that $*$ is a continuous $t$-norm, that

$$
\begin{aligned}
\lim _{n \rightarrow \infty} F_{x_{n}, x_{n+p}}(\varepsilon) \geq & {\left[\lim _{n \rightarrow \infty} F_{x_{n}, x_{n+1}}\left(\frac{\varepsilon}{p}\right)\right] *\left[\lim _{n \rightarrow \infty} F_{x_{n+1}, x_{n+2}}\left(\frac{\varepsilon}{p}\right)\right] * \cdots } \\
& *\left[\lim _{n \rightarrow \infty} F_{x_{n+p-1}, x_{n+p}}\left(\frac{\varepsilon}{p}\right)\right] \\
= & * 1 * \cdots * 1=1 .
\end{aligned}
$$

We have just proved that

$$
\lim _{n \rightarrow \infty} F_{x_{n}, x_{n+p}}(\varepsilon)=1 \quad \text { for all } \varepsilon>0 \text { and all } p \in \mathbb{N}^{*}
$$

which means that $\left\{x_{n}\right\}$ is a $G$-Cauchy sequence in $(X, F, *)$.

\section{Fixed point theorems in Menger probabilistic metric-like spaces}

In this section we present an extension of Theorem 1 in several ways: the probabilistic metric is more general, the contractivity condition is better and the involved auxiliary functions form a wider class.

Definition 16 We shall denote by $\mathcal{H}$ the family of all functions $h:(0,1] \rightarrow[0, \infty)$ satisfying:

$\left(\mathcal{H}_{1}\right)$ if $\left\{a_{n}\right\} \subset(0,1]$, then $\left\{a_{n}\right\} \rightarrow 1$ if, and only if, $\left\{h\left(a_{n}\right)\right\} \rightarrow 0$;

$\left(\mathcal{H}_{2}\right)$ if $\left\{a_{n}\right\} \subset(0,1]$, then $\left\{a_{n}\right\} \rightarrow 0$ if, and only if, $\left\{h\left(a_{n}\right)\right\} \rightarrow \infty$. 
The previous conditions are guaranteed when $h:(0,1] \rightarrow[0, \infty)$ is a strictly decreasing bijection between $(0,1]$ and $[0, \infty)$ such that $h$ and $h^{-1}$ are continuous (in a broad sense, it is sufficient to assume the continuities of $h$ and $h^{-1}$ on the extremes of the respective domains). For instance, this is the case of the function $h(t)=1 / t-1$ for all $t \in(0,1]$. However, the functions in $\mathcal{H}$ need not be continuous nor monotone.

Proposition 17 If $h \in \mathcal{H}$, then $h(1)=0$. Furthermore, $h(t)=0$ if, and only if, $t=1$.

Proof Using the sequence $a_{n}=1$ for all $n \in \mathbb{N}$ in $\left(\mathcal{H}_{1}\right)$, we deduce that $\left\{h(1)=h\left(a_{n}\right)\right\} \rightarrow 0$, so $h(1)=0$. On the other hand, assume that there exists $t \in(0,1]$ such that $h(t)=0$. If we define $a_{n}=t$ for all $n \in \mathbb{N}$, then $\left\{h\left(a_{n}\right)=0\right\} \rightarrow 0$. By condition $\left(\mathcal{H}_{1}\right),\left\{t=a_{n}\right\} \rightarrow 1$, so $t=1$.

The main result of the present manuscript is the following one.

Theorem 18 Let $(X, F, *)$ be a G-complete Menger PMLS and let $T: X \rightarrow X$ be a mapping. Suppose that there exist $c \in(0,1), \phi \in \Phi, \psi \in \Psi_{0}$, and $h \in \mathcal{H}$ such that

$$
h\left(F_{T x, T y}(\phi(c t))\right) \leq \psi\left(h\left(F_{x, y}(\phi(t))\right)\right)
$$

for all $x, y \in X$ and all $t>0$ for which $F_{x, y}(\phi(t))>0$. If there exists $x_{0} \in X$ such that $\lim _{t \rightarrow \infty} F_{x_{0}, T x_{0}}(t)=1$, then $T$ has at least one fixed point.

Additionally, assume that for all $x, y \in \operatorname{Fix}(T)$ with $x \neq y$, we have $\lim _{t \rightarrow \infty} F_{x y}(t)=1$. Then Thas a unique fixed point.

Notice that we shall not use the left-continuity of $\phi$ neither its strictly monotony. We shall only use that $\phi$ in nondecreasing. Hence, $\phi$ belongs to a more general class of auxiliary functions.

Proof Notice that condition (3) implies that if $F_{x, y}(\phi(t))>0$, then $h$ must be applicable to $F_{T x, T y}(\phi(c t))$. Hence $F_{T x, T y}(\phi(c t)) \in \operatorname{dom} h=(0,1]$, which means that

$$
F_{x, y}(\phi(t))>0 \Rightarrow F_{T x, T y}(\phi(c t))>0 .
$$

Let $x_{0} \in X$ be the point such that $F_{x_{0}, T x_{0}}\left(t_{x_{0}}\right)>0$, and let $\left\{x_{n}\right\}$ be the Picard sequence of $T$ based on $x_{0}$, that is, $x_{n+1}=T x_{n}$ for all $n \in \mathbb{N}$. If there exists some $n_{0} \in \mathbb{N}$ such that $x_{n_{0}}=x_{n_{0}+1}$, then $x_{n_{0}}$ is a fixed point of $T$, and the existence part of the proof is finished. On the contrary case, assume that $x_{n} \neq x_{n+1}$ for all $n \in \mathbb{N}$.

Since $\lim _{t \rightarrow \infty} F_{x_{0}, T x_{0}}(t)=1$, there exists $t_{0}>0$ such that $F_{x_{0}, x_{1}}\left(t_{0}\right)=F_{x_{0}, T x_{0}}\left(t_{0}\right)>0$. Moreover, as $\lim _{t \rightarrow \infty} \phi(t)=\infty$, it follows that there exists $s_{0} \in[0, \infty)$ (we can suppose, without loss of generality, that $\left.s_{0} \geq t_{0}\right)$ such that $\phi\left(s_{0}\right)>t_{0}$. Hence

$$
F_{x_{0} x_{1}}\left(\phi\left(s_{0}\right)\right) \geq F_{x_{0} x_{1}}\left(t_{0}\right)>0
$$

It follows from (4) that

$$
F_{x_{1} x_{2}}\left(\phi\left(c s_{0}\right)\right)=F_{T x_{0} T x_{1}}\left(\phi\left(c s_{0}\right)\right)>0,
$$


and, by induction, it can be proved that

$$
F_{x_{n} x_{n+1}}\left(\phi\left(c^{n} s_{0}\right)\right)>0 \quad \text { for all } n \in \mathbb{N} \text {. }
$$

If $n, m, r \in \mathbb{N}$ and $r \leq n$, then $c^{n} s_{0} \leq c^{r} s_{0} \leq s_{0} \leq \frac{s_{0}}{c^{m}}$. Since $\phi$ and $F_{x_{n} x_{n+1}}$ are nondecreasing functions, it follows that

$$
\begin{aligned}
& \text { if } n, m, r \in \mathbb{N} \text { and } r \leq n \text {, then } \\
& \begin{aligned}
0 & <F_{x_{n} x_{n+1}}\left(\phi\left(c^{n} s_{0}\right)\right) \leq F_{x_{n} x_{n+1}}\left(\phi\left(c^{r} s_{0}\right)\right) \leq F_{x_{n} x_{n+1}}\left(\phi\left(s_{0}\right)\right) \\
& \leq F_{x_{n} x_{n+1}}\left(\phi\left(\frac{s_{0}}{c^{m}}\right)\right) .
\end{aligned}
\end{aligned}
$$

We claim that

$$
\lim _{n \rightarrow \infty} F_{x_{n}, x_{n+1}}(s)=1 \quad \text { for all } s>0 .
$$

In order to prove it, let $s>0$ be arbitrary. As $\lim _{r \rightarrow \infty}\left(c^{r} s_{0}\right)=0$ and $\phi$ is continuous at $t=0$, then $\lim _{r \rightarrow \infty} \phi\left(c^{r} s_{0}\right)=\phi(0)=0$. Since $s>0$, there exists $r \in \mathbb{N}$ such that

$$
\phi\left(c^{r} s_{0}\right) \leq s
$$

Let $n \in \mathbb{N}$ be such that $n>r$. Applying the contractivity condition (3) to $x=x_{n}$ and $y=x_{n+1}$, it follows that

$$
\begin{aligned}
h\left(F_{x_{n}, x_{n+1}}\left(\phi\left(c^{r} s_{0}\right)\right)\right) & =h\left(F_{T x_{n-1}, T x_{n}}\left(\phi\left(c^{r} s_{0}\right)\right)\right) \\
& \leq \psi\left(h\left(F_{x_{n-1}, x_{n}}\left(\phi\left(c^{r-1} s_{0}\right)\right)\right)\right),
\end{aligned}
$$

where we have used $F_{x_{n-1}, x_{n}}\left(\phi\left(c^{r-1} s_{0}\right)\right)>0$ by (5). Repeating this argument, we find that

$$
\begin{aligned}
h\left(F_{x_{n-1}, x_{n}}\left(\phi\left(c^{r-1} s_{0}\right)\right)\right) & =h\left(F_{T x_{n-2}, T x_{n-1}}\left(\phi\left(c^{r-1} s_{0}\right)\right)\right) \\
& \leq \psi\left(h\left(F_{x_{n-2}, x_{n-1}}\left(\phi\left(c^{r-2} s_{0}\right)\right)\right)\right),
\end{aligned}
$$

where we have used $F_{x_{n-2}, x_{n-1}}\left(\phi\left(c^{r-2} s_{0}\right)\right)>0$ by (5). As $\psi$ is nondecreasing, then

$$
\psi\left(h\left(F_{x_{n-1}, x_{n}}\left(\phi\left(c^{r-1} s_{0}\right)\right)\right)\right) \leq \psi^{2}\left(h\left(F_{x_{n-2}, x_{n-1}}\left(\phi\left(c^{r-2} s_{0}\right)\right)\right)\right) .
$$

Combining inequalities (8) and (9), we deduce that

$$
\begin{aligned}
h\left(F_{x_{n}, x_{n+1}}\left(\phi\left(c^{r} s_{0}\right)\right)\right) & \leq \psi\left(h\left(F_{x_{n-1}, x_{n}}\left(\phi\left(c^{r-1} s_{0}\right)\right)\right)\right) \\
& \leq \psi^{2}\left(h\left(F_{x_{n-2}, x_{n-1}}\left(\phi\left(c^{r-2} s_{0}\right)\right)\right)\right) .
\end{aligned}
$$

Inequalities (5) permit us to repeat this argument $n$ times, and it follows that

$$
h\left(F_{x_{n}, x_{n+1}}\left(\phi\left(c^{r} s_{0}\right)\right)\right) \leq \psi^{n}\left(h\left(F_{x_{0}, x_{1}}\left(\phi\left(c^{r-n} s_{0}\right)\right)\right)\right)=\psi^{n}\left(h\left(F_{x_{0}, x_{1}}\left(\phi\left(\frac{s_{0}}{c^{n-r}}\right)\right)\right)\right),
$$


for all $n>r$. As a consequence,

$$
\begin{aligned}
\lim _{n \rightarrow \infty} \frac{s_{0}}{c^{n-r}}=\infty & \Rightarrow \lim _{n \rightarrow \infty} \phi\left(\frac{s_{0}}{c^{n-r}}\right)=\infty \\
& \Rightarrow \lim _{n \rightarrow \infty} F_{x_{0}, x_{1}}\left(\phi\left(\frac{s_{0}}{c^{n-r}}\right)\right)=1 \\
& \Rightarrow \lim _{n \rightarrow \infty} h\left(F_{x_{0}, x_{1}}\left(\phi\left(\frac{s_{0}}{c^{n-r}}\right)\right)\right)=0 .
\end{aligned}
$$

As the sequence $\left\{a_{n}=h\left(F_{x_{0}, x_{1}}\left(\phi\left(\frac{s_{0}}{c^{n-r}}\right)\right)\right)\right\} \rightarrow 0$ and $h \in \mathcal{H}$, we have $\left\{\psi^{n}\left(a_{n}\right)\right\} \rightarrow 0$. By (10), we deduce that

$$
\lim _{n \rightarrow \infty} h\left(F_{x_{n}, x_{n+1}}\left(\phi\left(c^{r} s_{0}\right)\right)\right)=0 .
$$

In particular, as $h \in \mathcal{H}$, condition $\left(\mathcal{H}_{1}\right)$ implies that

$$
\lim _{n \rightarrow \infty} F_{x_{n}, x_{n+1}}\left(\phi\left(c^{r} s_{0}\right)\right)=1 .
$$

Taking into account (7), we observe that

$$
F_{x_{n}, x_{n+1}}\left(\phi\left(c^{r} s_{0}\right)\right) \leq F_{x_{n}, x_{n+1}}(s) \leq 1 .
$$

Therefore,

$$
\lim _{n \rightarrow \infty} F_{x_{n}, x_{n+1}}(s)=1,
$$

which means that (6) holds. Lemma 15 guarantees that $\left\{x_{n}\right\}$ is a G-Cauchy sequence in $(X, F, *)$. As it is $G$-complete, there exists $z \in X$ such that $\left\{x_{n}\right\} \rightarrow z$, that is,

$$
\forall \varepsilon>0, \forall \lambda \in(0,1), \exists n_{0} \in \mathbb{N}:\left(F_{x_{n} z}(\varepsilon)>1-\lambda, \forall n \geq n_{0}\right) .
$$

We claim that $z$ is a fixed point of $T$. To prove it, observe that, for all $t>0$ and all $n \in \mathbb{N}$,

$$
\begin{aligned}
F_{z, T z}(t) & \geq F_{z, x_{n+1}}\left(\frac{t}{2}\right) * F_{x_{n+1}, T z}\left(\frac{t}{2}\right) \\
& =F_{z, x_{n+1}}\left(\frac{t}{2}\right) * F_{T x_{n}, T z}\left(\frac{t}{2}\right) .
\end{aligned}
$$

By (11), it is clear that

$$
\lim _{n \rightarrow \infty} F_{z, x_{n}}(s)=1 \quad \text { for all } s>0 .
$$

In particular,

$$
\lim _{n \rightarrow \infty} F_{z, x_{n+1}}\left(\frac{t}{2}\right)=1
$$


Let us show that the second factor in (12) also converges to 1 when $n$ tends to $\infty$. Taking into account that $\phi$ is continuous at $t=0$, we have $\lim _{s \rightarrow 0} \phi(s)=\phi(0)=0$. Since $t / 2>0$, there exists $\delta>0$ such that $\phi(\delta)<t / 2$. Since $\delta / c>0, \phi(\delta / c)>0$. By (13),

$$
\lim _{n \rightarrow \infty} F_{x_{n}, z}\left(\phi\left(\frac{\delta}{c}\right)\right)=1
$$

Hence, there exists $n_{0} \in \mathbb{N}$ such that

$$
F_{x_{n}, z}\left(\phi\left(\frac{\delta}{c}\right)\right)>0 \quad \text { for all } n \geq n_{0} .
$$

Applying the contractivity condition (3) to $x=z$ and $y=x_{n+1}$ for $n \geq n_{0}$, we observe that

$$
h\left(F_{x_{n+1}, T z}(\phi(\delta))\right)=h\left(F_{T x_{n}, T z}(\phi(\delta))\right) \leq \psi\left(h\left(F_{x_{n}, z}\left(\phi\left(\frac{\delta}{c}\right)\right)\right)\right) .
$$

Therefore

$$
\begin{aligned}
& \lim _{n \rightarrow \infty} F_{x_{n}, z}\left(\phi\left(\frac{\delta}{c}\right)\right)=1 \\
& \Rightarrow \quad \lim _{n \rightarrow \infty} h\left(F_{x_{n}, z}\left(\phi\left(\frac{\delta}{c}\right)\right)\right)=0 \\
& \Rightarrow \quad \lim _{n \rightarrow \infty} \psi\left(h\left(F_{x_{n}, z}\left(\phi\left(\frac{\delta}{c}\right)\right)\right)\right)=0 \\
& \Rightarrow \quad \lim _{n \rightarrow \infty} h\left(F_{x_{n+1}, T z}(\phi(\delta))\right)=0 \\
& \Rightarrow \quad \lim _{n \rightarrow \infty} F_{x_{n+1}, T z}(\phi(\delta))=1 .
\end{aligned}
$$

Taking into account that

$$
F_{x_{n+1}, T z}(\phi(\delta)) \leq F_{x_{n+1}, T z}\left(\frac{t}{2}\right) \leq 1,
$$

we deduce that

$$
\lim _{n \rightarrow \infty} F_{T x_{n}, T z}\left(\frac{t}{2}\right)=\lim _{n \rightarrow \infty} F_{x_{n+1}, T z}\left(\frac{t}{2}\right)=1 .
$$

Letting $n \rightarrow \infty$ in (12) and using (14) and (15), we deduce that

$$
\begin{aligned}
F_{z, T z}(t) & \geq \lim _{n \rightarrow \infty}\left[F_{z, x_{n+1}}\left(\frac{t}{2}\right) * F_{T x_{n}, T z}\left(\frac{t}{2}\right)\right] \\
& =\left[\lim _{n \rightarrow \infty} F_{z, x_{n+1}}\left(\frac{t}{2}\right)\right] *\left[\lim _{n \rightarrow \infty} F_{T x_{n}, T z}\left(\frac{t}{2}\right)\right]=1 * 1=1 .
\end{aligned}
$$

We have just proved that $F_{z, T z}(t)=1$ for all $t>0$, and axiom $\left(\mathrm{PM}_{2}\right)$ guarantees that $T z=z$, that is, $z$ is a fixed point of $T$.

Next, we study the uniqueness of the fixed point of $T$. Assume that $T$ has two different fixed points $x$ and $y$, and we will obtain the contradiction $x=y$. By hypothesis, 
$\lim _{t \rightarrow \infty} F_{x y}(t)=1$. Then there exists $t_{0}>0$ such that $F_{x y}\left(t_{0}\right)>0$. Moreover, there exists $s_{0}>0$ such that

$$
\phi\left(s_{0}\right)>t_{0}
$$

Consequently, as $\phi$ and $F_{x y}$ are nondecreasing functions,

$$
F_{x y}\left(\phi\left(s_{0}\right)\right) \geq F_{x y}\left(t_{0}\right)>0 .
$$

By (4), we have

$$
F_{x y}\left(\phi\left(s_{0}\right)\right)>0 \Rightarrow F_{x y}\left(\phi\left(c s_{0}\right)\right)=F_{T x, T y}\left(\phi\left(c s_{0}\right)\right)>0 .
$$

By induction,

$$
F_{x y}\left(\phi\left(c^{n} s_{0}\right)\right)>0 \quad \text { for all } n \in \mathbb{N} \text {. }
$$

We claim that

$$
F_{x y}\left(\phi\left(c^{r} s_{0}\right)\right)=1 \quad \text { for all } r \in \mathbb{N} \text {. }
$$

To prove it, let $r \in \mathbb{N}$ be arbitrary and let $n, m \in \mathbb{N}$ be such that $n>r$. As $c^{n} s_{0} \leq c^{r} s_{0} \leq s_{0} \leq$ $\frac{s_{0}}{c^{m}}$, and $\phi$ and $F_{x y}$ are nondecreasing functions, it follows that

$$
\begin{aligned}
& \text { if } n, m \in \mathbb{N} \text { and } r \leq n \text {, then } \\
& 0<F_{x y}\left(\phi\left(c^{n} s_{0}\right)\right) \leq F_{x y}\left(\phi\left(c^{r} s_{0}\right)\right) \leq F_{x y}\left(\phi\left(s_{0}\right)\right) \leq F_{x y}\left(\phi\left(\frac{s_{0}}{c^{m}}\right)\right) .
\end{aligned}
$$

Applying the contractivity condition (3) to $x$ and $y$, it follows that

$$
h\left(F_{x y}\left(\phi\left(c^{r} s_{0}\right)\right)\right)=h\left(F_{T x, T y}\left(\phi\left(c^{r} s_{0}\right)\right)\right) \leq \psi\left(h\left(F_{x y}\left(\phi\left(c^{r-1} s_{0}\right)\right)\right)\right),
$$

where we have used $F_{x y}\left(\phi\left(c^{r-1} s_{0}\right)\right)>0$ by (17). Repeating this argument, we find that

$$
h\left(F_{x y}\left(\phi\left(c^{r-1} s_{0}\right)\right)\right)=h\left(F_{T x, T y}\left(\phi\left(c^{r-1} s_{0}\right)\right)\right) \leq \psi\left(h\left(F_{x y}\left(\phi\left(c^{r-2} s_{0}\right)\right)\right)\right),
$$

where we have used $F_{x y}\left(\phi\left(c^{r-2} s_{0}\right)\right)>0$ by (17). As $\psi$ is nondecreasing, we have

$$
\psi\left(h\left(F_{x y}\left(\phi\left(c^{r-1} s_{0}\right)\right)\right)\right) \leq \psi^{2}\left(h\left(F_{x y}\left(\phi\left(c^{r-2} s_{0}\right)\right)\right)\right)
$$

Combining inequalities (18) and (19), we deduce that

$$
h\left(F_{x y}\left(\phi\left(c^{r} s_{0}\right)\right)\right) \leq \psi\left(h\left(F_{x y}\left(\phi\left(c^{r-1} s_{0}\right)\right)\right)\right) \leq \psi^{2}\left(h\left(F_{x y}\left(\phi\left(c^{r-2} s_{0}\right)\right)\right)\right) .
$$

Inequalities (17) permit us to repeat this argument $n$ times, and it follows that

$$
h\left(F_{x y}\left(\phi\left(c^{r} s_{0}\right)\right)\right) \leq \psi^{n}\left(h\left(F_{x y}\left(\phi\left(c^{r-n} s_{0}\right)\right)\right)\right)=\psi^{n}\left(h\left(F_{x y}\left(\phi\left(\frac{s_{0}}{c^{n-r}}\right)\right)\right)\right)
$$


for all $n>r$. As a consequence,

$$
\begin{aligned}
\lim _{n \rightarrow \infty} \frac{s_{0}}{c^{n-r}}=\infty & \Rightarrow \lim _{n \rightarrow \infty} \phi\left(\frac{s_{0}}{c^{n-r}}\right)=\infty \\
& \Rightarrow \lim _{n \rightarrow \infty} F_{x y}\left(\phi\left(\frac{s_{0}}{c^{n-r}}\right)\right)=1 \\
& \Rightarrow \lim _{n \rightarrow \infty} h\left(F_{x y}\left(\phi\left(\frac{s_{0}}{c^{n-r}}\right)\right)\right)=0 .
\end{aligned}
$$

As the sequence $\left\{a_{n}=h\left(F_{x y}\left(\phi\left(\frac{s_{0}}{c^{n-r}}\right)\right)\right)\right\} \rightarrow 0$ and $h \in \mathcal{H}$, we have $\left\{\psi^{n}\left(a_{n}\right)\right\} \rightarrow 0$. By (20), we deduce that

$$
h\left(F_{x y}\left(\phi\left(c^{r} s_{0}\right)\right)\right)=0 .
$$

In particular, as $h \in \mathcal{H}$, Proposition 17 implies that

$$
F_{x y}\left(\phi\left(c^{r} s_{0}\right)\right)=1
$$

which means that (16) holds. Next, let us show that $F_{x y}(t)=1$ for all $t>0$. Let $t>0$ arbitrary. Since $\lim _{n \rightarrow \infty}\left(c^{n} s_{0}\right)=0$ and $\lim _{n \rightarrow \infty} \phi\left(c^{n} s_{0}\right)=\phi(0)=0$, there exists $r \in \mathbb{N}$ such that $\phi\left(c^{r} s_{0}\right)<t$. Hence

$$
1=F_{x y}\left(\phi\left(c^{r} s_{0}\right)\right) \leq F_{x y}(t)=1,
$$

so $F_{x y}(t)=1$. Varying $t>0$, we conclude that $x=y$ by virtue of $\left(\mathrm{PM}_{2}\right)$, which contradicts the fact that $x \neq y$. As a result, $T$ can only have a unique fixed point.

In the following result, condition $\left(\mathrm{PM}_{5}\right)$ guarantees that any initial condition $x_{0} \in X$ yields a fixed point.

Corollary 19 Let $(X, F, *)$ be a G-complete Menger PMLS verifying $\left(\mathrm{PM}_{5}\right)$ and let $T: X \rightarrow$ $X$ be a mapping. Suppose that there exist $c \in(0,1), \phi \in \Phi, \psi \in \Psi_{0}$, and $h \in \mathcal{H}$ such that

$$
h\left(F_{T x, T y}(\phi(c t))\right) \leq \psi\left(h\left(F_{x, y}(\phi(t))\right)\right)
$$

for all $x, y \in X$ and all $t>0$ for which $F_{x, y}(\phi(t))>0$. Then $T$ has a unique fixed point.

The previous results are also valid for Menger PM-spaces.

Corollary 20 Let $(X, F, *)$ be a G-complete Menger PMS and let $T: X \rightarrow X$ be a mapping. Suppose that there exist $c \in(0,1), \phi \in \Phi, \psi \in \Psi_{0}$, and $h \in \mathcal{H}$ such that

$$
h\left(F_{T x, T y}(\phi(c t))\right) \leq \psi\left(h\left(F_{x, y}(\phi(t))\right)\right)
$$

for all $x, y \in X$ and all $t>0$ for which $F_{x, y}(\phi(t))>0$. If there exists $x_{0} \in X$ such that $\lim _{t \rightarrow \infty} F_{x_{0}, T x_{0}}(t)=1$, then $T$ has at least one fixed point.

Additionally, assume that for all $x, y \in \operatorname{Fix}(T)$ with $x \neq y$, we have $\lim _{t \rightarrow \infty} F_{x y}(t)=1$. Then $T$ has a unique fixed point. 
Corollary 21 Let $(X, F, *)$ be a G-complete Menger PMS verifying $\left(\mathrm{PM}_{5}\right)$ and let $T: X \rightarrow X$ be a mapping. Suppose that there exist $c \in(0,1), \phi \in \Phi, \psi \in \Psi_{0}$, and $h \in \mathcal{H}$ such that

$$
h\left(F_{T x, T y}(\phi(c t))\right) \leq \psi\left(h\left(F_{x, y}(\phi(t))\right)\right)
$$

for all $x, y \in X$ and all $t>0$ for which $F_{x, y}(\phi(t))>0$. Then $T$ has a unique fixed point.

The following statement trivially follows from Theorem 18 using $h(t)=1 / t-1$ for all $t \in(0,1]$.

Corollary 22 Theorem 1 immediately follows from Theorem 18.

Example 23 Let $\left(X, F, *_{P}\right)$ be the Menger PMLS introduced in Example 9 and let $T: X \rightarrow$ $X$ be the self-mapping defined by $T x=x / 2$ for all $x \in X$. We know that $\left(X, F, *_{P}\right)$ is a Menger PMLS that also verifies $\left(\mathrm{PM}_{5}\right)$, but it is not a Menger PMS. In Example 12 we showed that $\left(X, F, *_{P}\right)$ is $G$-complete. Assume that $\psi(t)=\phi(t)=t$ for all $t \in[0, \infty)$, and let $h$ : $(0,1] \rightarrow[0, \infty)$ be whatever strictly decreasing bijection between $(0,1]$ and $[0, \infty)$ such that $h$ and $h^{-1}$ are continuous (for instance, $h(t)=1 / t-1$ for all $t \in(0,1]$, but any other function verifying these properties yields the same result). In this context, the contractivity condition (3) is equivalent to

$$
\begin{gathered}
h\left(F_{T x, T y}(\phi(c t))\right) \leq \psi\left(h\left(F_{x, y}(\phi(t))\right)\right) \\
\Leftrightarrow \quad h\left(F_{T x, T y}(c t)\right) \leq h\left(F_{x, y}(t)\right) \\
\Leftrightarrow \quad F_{T x, T y}(c t) \geq F_{x, y}(t) .
\end{gathered}
$$

If $c=1 / 2$, then, for all $t>0$,

$$
\begin{aligned}
F_{T x, T y}(c t) & =F_{x / 2, y / 2}\left(\frac{t}{2}\right)=F_{x / 2, y / 2}\left(\frac{t}{2}\right)=\epsilon_{\max \{x / 2, y / 2\}}\left(\frac{t}{2}\right) \\
& =\left\{\begin{array}{ll}
0, & \text { if } 0 \leq \frac{t}{2} \leq \max \left\{\frac{x}{2}, \frac{y}{2}\right\}, \\
1, & \text { if } \frac{t}{2}>\max \left\{\frac{x}{2}, \frac{y}{2}\right\}
\end{array}\right\} \\
& =\left\{\begin{array}{ll}
0, & \text { if } 0 \leq t \leq \max \{x, y\}, \\
1, & \text { if } t>\max \{x, y\}
\end{array}\right\} \\
& =\epsilon_{\max \{x, y\}}(t)=F_{x, y}(t) .
\end{aligned}
$$

As a result, the contractivity condition is verified. Hence, Corollary 19 guarantees that $T$ has a unique fixed point (which is $z=0$ ). Notice that other previous results are not applicable to this example because $\left(X, F, *_{P}\right)$ is not a Menger PMS. 


\section{Author details}

${ }^{1}$ Department of Quantitative Methods for Economics and Business, Faculty of Economics and Business Sciences, University of Granada, Campus Universitario de Cartuja, s/n, Granada, 18011, Spain. ${ }^{2}$ Institute of Research and Development of Processes, Faculty of Science and Technology, University of Basque Country, Campus of Leioa, Barrio Sarriena, Leioa (Bizkaia), 48940, Spain.

\section{Acknowledgements}

The authors are very thankful to the anonymous reviewers for their careful reading of this manuscript and for their constructive reports, which have been very useful to improve the paper. M de la Sen is grateful to the Spanish Government for its support of this research through Grant DPI2012-30651 and to the Basque Government for its support of this research through Grants IT378-10 and SAIOTEK S-PE12UN015. He is also grateful to the University of Basque Country for its financial support through Grant UFI 2011/07. A-F Roldán-López-de-Hierro has been partially supported by Junta de Andalucía by project FQM-268 of the Andalusian CICYE. He is also grateful to the Department of Quantitative Methods for Economics and Business of the University of Granada for its support.

\section{Endnote}

a At present, a cumulative distribution function is usually defined as probability of a variable which is less than or equal to $t$, not less than $t$; since Menger's work in probabilistic spaces, it is commonly used the strict inequality.

Received: 18 May 2015 Accepted: 8 September 2015 Published online: 29 September 2015

\section{References}

1. Zimmerman, HJ: Fuzzy Set Theory and Its Application, 3rd edn. Kluwer Academic, Boston (1991)

2. Kramosil, I, Michálek, J: Fuzzy metrics and statistical metric spaces. Kybernetika 11, 336-344 (1975)

3. Erceg, MA: Metric spaces in fuzzy set theory. J. Math. Anal. Appl. 69, 205-230 (1979)

4. Deng, Z: Fuzzy pseudo metric spaces. J. Math. Anal. Appl. 86, 74-95 (1982)

5. Kaleva, O, Seikkala, S: On fuzzy metric spaces. Fuzzy Sets Syst. 12, 215-229 (1984)

6. George, A, Veeramani, P: On some results in fuzzy metric spaces. Fuzzy Sets Syst. 64, 395-399 (1994)

7. Roldán, A, Martínez-Moreno, J, Roldán, C: On interrelationships between fuzzy metric structures. Iran. J. Fuzzy Syst. 10, 133-150 (2013)

8. Schweizer, B, Sklar, A: Probabilistic Metric Spaces. Dover, New York (2005)

9. Grabiec, M, Cho, YJ, Radu, V: On Nonsymmetric Topological and Probabilistic Structures. Nova Science Publishers, New York (2006)

10. Hadžić, O, Pap, E: Fixed Point Theory in Probabilistic Metric Spaces. Kluwer Academic, Dordrecht (2001)

11. Chang, S-S, Cho, YJ, Kang, SM: Nonlinear Operator Theory in Probabilistic Metric Spaces. Nova Science Publishers, New York (2001)

12. Kutbi, MA, Gopal, D, Vetro, C, Sintunaravat, W: Further generalization of fixed point theorems in Menger PM-spaces. Fixed Point Theory Appl. 2015, Article ID 32 (2015)

13. Gregori, V, Sapena, A: On fixed-point theorems in fuzzy metric spaces. Fuzzy Sets Syst. 125, 245-252 (2002)

14. Mihet, D: On fuzzy contractive mappings in fuzzy metric spaces. Fuzzy Sets Syst. 158, 915-921 (2007)

15. Mihet, D: Fuzzy $\psi$-contractive mappings in non-Archimedean fuzzy metric spaces. Fuzzy Sets Syst. 159, 739-744 (2008)

16. Gopal, D, Vetro, C: Some new fixed point theorems in fuzzy metric spaces. Iran. J. Fuzzy Syst. 11(3), $95-107$ (2014)

17. Shukla, S, Abbas, M: Fixed point results in fuzzy metric-like spaces. Iran. J. Fuzzy Syst. 11(5), 81-92 (2014)

18. Roldán-López-de-Hierro, AF, Karapınar, E, Manro, S: Some new fixed point theorems in fuzzy metric spaces. J. Intell. Fuzzy Syst. 27, 2257-2264 (2014)

19. Roldán-López-de-Hierro, AF, Karapınar, E, Kumam, P: Irremissible stimulate on 'Unified fixed point theorems in fuzzy metric spaces via common limit range property'. J. Inequal. Appl. 2014, Article ID 257 (2014)

\section{Submit your manuscript to a SpringerOpen ${ }^{\circ}$ journal and benefit from:}

- Convenient online submission

Rigorous peer review

- Immediate publication on acceptance

- Open access: articles freely available online

- High visibility within the field

- Retaining the copyright to your article 